\title{
Logarithmic nonlinear Schrödinger equation and irrotational, compressible flows: An exact solution
}

\author{
K. W. Chow* \\ Department of Mechanical Engineering, University of Hong Kong, Pokfulam, Hong Kong \\ (Received 14 February 2011; revised manuscript received 12 May 2011; published 14 July 2011)
}

\begin{abstract}
A class of irrotational, isentropic, and compressible flows is studied theoretically by formulating the density and the velocity potential in a Madelung transformation. The resulting nonlinear Schrödinger equation is solved in terms of similarity variables. One particular family of exact solutions, valid for any ratio of the specific heat capacities of the gas, permits explicit expressions of the fluid properties and velocities in terms of time and spatial coordinates. Analytically, the density is a Gaussian function of the similarity variable, while the temperature is a function of time only. This method is applicable in one (1D), two, and three dimensional geometries. As a simple example, a 1D gas column, with mass injection on one side and a steadily translating wall on the other, can be formulated exactly. The connection with the evolution of an unsteady velocity potential will also be examined.
\end{abstract}

DOI: 10.1103/PhysRevE.84.016308

PACS number(s): 47.40.Dc, 47.15.km, 02.30.Hq, 02.30.Jr

\section{INTRODUCTION}

The irrotational flow of an inviscid, incompressible fluid is a classical subject, as the zero vorticity assumption automatically satisfies the nonlinear equations of motion through the Bernoulli equation. Conservation of mass then implies the study of the Laplace equation [1]. Applications and flow physics associated with such flows have been investigated $[2,3]$. For a compressible fluid, the dynamics is more complex, as the governing equations are highly nonlinear, even for irrotational flows [1]. If compact or extended distributions of vorticity are present in the compressible flow, the analysis becomes exceedingly intriguing [4-7].

The objective here is to deduce a special class of exact expressions of irrotational, compressible flows, by employing a Madelung transformation of the density and the velocity potential. The resulting nonlinear Schrödinger equation (NLSE), with logarithmic as well as Bohm-type nonlinearities, are solved by similarity variables. The intermediate calculations involve a set of nonlinear ordinary differential equations, which will reduce to the classical Ermakov system for the integrable case of a monatomic gas.

The present algorithm is applicable to one (1D), two, and three dimensional gas dynamics. As physical examples, in three dimensions, this family of flows will represent an expanding gas cloud. In 1D, a gas column, with mass injection on one side and a steadily translating wall on the other, can be described analytically. In two dimensional flows, the same analytical formulation works, and the connection with classical fluid mechanics will be highlighted. The capability of the scheme will be vividly demonstrated by showing that the velocity potential from this algorithm will satisfy a highly nonlinear evolution equation for unsteady, irrotational, and compressible flows.

The NLSE with logarithmic nonlinearity only, i.e., the one without the Bohm type, has an established history. Localized solutions in such logarithmic NLSEs, termed gaussons, have been discovered [8]. Applications have been pursued actively,

*kwchow@hku.hk especially in the context of fluid dynamics [9], magma transport [10], nuclear physics [11], and optics [12,13].

The structure of the paper can now be explained. The motion of an irrotational, compressible fluid is first formulated in terms of a NLSE with modulated logarithmic and Bohm-type nonlinearities. The modulation is due to the time dependent temperature of the gas. Requiring the flow to be devoid of vorticity, the assumption of similarity variables will then dictate the dynamics to be governed by a set of nonlinear, ordinary differential equations (ODEs).

Many earlier works, perhaps through different formulations, have focused on the integrable case, which applies to a monatomic gas only (Sec. II). In such cases, these autonomous ODEs reduce to Ermakov systems, where the dependent variables occur in special rational expressions. The distinguished feature is the admittance of an integral of motion, namely, the Ray-Reid invariant [14-16]. If, in addition, the system is Hamiltonian, then explicit integration will be feasible.

The main contribution here is a special class of analytic solutions, valid for any ratio of the specific heat capacities of the gas and, thus, not necessarily restricted to the integrable case of monatomic gas only [17]. Another merit of the present solution is that the density will be a Gaussian function of the similarity variable and appears to be different from all existing results.

In terms of flow configuration, these solutions correspond to a Gaussian function of density, a temperature field dependent on time only, and a velocity linear in the spatial coordinates. These analytical expressions permit a detailed analysis of the fluid physics. The general three dimensional setting, in terms of an expanding gas sphere, is explained first. The fluid properties, e.g., mass flux and Mach number, are delineated (Sec. III). Reductions to two and 1D geometries are outlined, and solving a highly nonlinear partial differential equation for the unsteady velocity potential by this formulation is discussed (Sec. IV). The special case of an expanding, 1D gas column with mass injection is derived (Sec. V). Precise comparisons with, and differences from, earlier works in the literature are given in Sec. IV. 


\section{FORMULATION}

The motion of a compressible fluid is governed by the conservation laws of mass, momentum, and energy. The first two principles, written with standard notations ( $\boldsymbol{q}$ being the velocity vector), are

$$
\begin{gathered}
\frac{\partial \rho}{\partial t}+\operatorname{div}(\rho \boldsymbol{q})=0, \\
\frac{\partial \boldsymbol{q}}{\partial t}+(\boldsymbol{q} \cdot \nabla) \boldsymbol{q}=-\frac{\nabla p}{\rho} .
\end{gathered}
$$

The energy balance is accounted for through a search for flows with constant entropy. Nevertheless, the exact solutions developed in this paper will be shown to satisfy a conventional form of the energy equation to be discussed in further detail in Sec. III.

The entropy $S$ is calculated from ( $\gamma$ being the ratio of the specific heat capacities)

$$
S=-\ln \rho+\frac{1}{\gamma-1} \ln T, \quad \gamma \neq 1 .
$$

We now look for isentropic flows where $S$ is conserved. The material derivative of the entropy vanishes,

$$
\frac{\partial S}{\partial t}+\boldsymbol{q} \cdot \nabla S=0 .
$$

We also assume an ideal gas law and adopt scaled coordinates such that the gas constant is unity, i.e.,

$$
p=\rho T .
$$

We will attempt to produce a special class of exact solutions for compressible flows satisfying Eqs. (1)-(5).

To begin, the continuity equation can also be rewritten as

$$
\operatorname{div} \boldsymbol{q}=-\frac{1}{\gamma-1} \frac{D}{D t} \ln T, \quad \gamma \neq 1
$$

where the material derivative is

$$
\frac{D}{D t}=\frac{\partial}{\partial t}+(\boldsymbol{q} \cdot \nabla)
$$

Likewise, the momentum equation can be cast in two equivalent forms

$$
\begin{gathered}
\frac{\partial \boldsymbol{q}}{\partial t}+(\boldsymbol{q} \cdot \nabla) \boldsymbol{q}=T \nabla S-\frac{\gamma}{\gamma-1} \nabla T, \\
\text { or } \quad \frac{\partial \boldsymbol{q}}{\partial t}+(\boldsymbol{q} \cdot \nabla) \boldsymbol{q}+T \nabla \ln \rho+\nabla T=0 .
\end{gathered}
$$

One now looks for special configurations where the temperature $T$ is a function of time $t$ only, i.e., independent of $x$, $y$, and $z$. Furthermore, we seek irrotational flows where the velocity vector is a gradient of a scalar potential $\Phi$,

$$
\boldsymbol{q}=\nabla \Phi
$$

The Madelung transformation,

$$
\Psi=\rho^{1 / 2} \exp \left[i \frac{\Phi}{2}\right]
$$

reduces the whole formulation to the nonlinear Schrödinger equation,

$$
i \frac{\partial \Psi}{\partial t}+\nabla^{2} \Psi-T(t)(\ln |\Psi|) \Psi-\left(\frac{\nabla^{2}|\Psi|}{|\Psi|}\right) \Psi=0,
$$

a NLSE with both Bialynicki-Birula- (logarithmic) type and de Broglie-Bohm-type potential terms for the complex envelope $\Psi$. Here, the logarithmic potential is being modulated in time, since the temperature will fluctuate as the gas flows.

Motivated by the gausson of the logarithmic NLSE [8], one searches for an irrotational velocity field $(\mathbf{i}, \mathbf{j}$, and $\mathbf{k}$ are unit vectors in the $x, y$, and $z$ directions),

$$
\begin{aligned}
\boldsymbol{q}= & \left\{\frac{\dot{a}(t)}{a(t)}[x-\lambda(t)]+\dot{\lambda}(t)\right\} \mathbf{i}+\left\{\frac{\dot{b}(t)}{b(t)}[y-\mu(t)]+\dot{\mu}(t)\right\} \mathbf{j} \\
& +\left\{\frac{\dot{c}(t)}{c(t)}[z-\theta(t)]+\dot{\theta}(t)\right\} \mathbf{k},
\end{aligned}
$$

together with a density given by

$\rho=\sigma(t) \exp \left\{+\frac{[x-\lambda(t)]^{2}}{a^{2}(t)}+\frac{[y-\mu(t)]^{2}}{b^{2}(t)}+\frac{[z-\theta(t)]^{2}}{c^{2}(t)}\right\}$.

While an exponentially decreasing density field seems more natural, the particular solution we study below will lead to a negative temperature with such density. The increasing exponential function of Eq. (12) will imply that this solution will be restricted to a finite domain only to avoid the problem of an infinite mass.

\section{A. Insight from fluid mechanics}

A remark on the justification and usage of a Gaussian profile in Eq. (12), from the viewpoint of fluid mechanics, might be helpful. From classical fluid dynamics, the mathematical structures of gas dynamics equations and those of shallow water waves are similar. Indeed, velocity profiles as linear expressions of spatial coordinates multiplied by functions of time have been used in shallow water waves a long time ago [18]. The present paper attempts to extend this reasoning to gas dynamics. The density must then be Gaussian, and the algebra can vividly be demonstrated for the simple case of 1D steady barotropic flow $\left(G_{1}, G_{2}=\right.$ functions, $c_{\text {sound }}=$ speed of sound, and $\left.c_{\text {sound }}^{2}=d p / d \rho[19,20]\right)$,

$$
u u_{x}=-\frac{c_{\text {sound }}^{2} \rho_{x}}{\rho} .
$$

If a linear expression in $x$,

$$
u=G_{1}(t) x
$$

is assumed, then it follows immediately that

$$
\frac{\rho_{x}}{\rho}=G_{2}(t) x
$$

if $c_{\text {sound }}$ depends on time only, as in the present case, and thus,

$$
\rho \sim \exp \left[\frac{G_{2}(t) x^{2}}{2}\right] .
$$

In fact, a similar expansion format has also been used in treating a harmonic oscillator [21]. 
Returning now to the analytical formulation, mass conservation (1) and Eqs. (11) and (12) now show that

$$
\sigma=\frac{C_{1}}{a b c},
$$

where $C_{n}, n$ as an integer, will denote constants in this paper. If the temperature field depends on time $t$ only, Eq. (6) will imply

$$
(a b c)^{\gamma-1}=\frac{C_{2}}{T} .
$$

The next step is the consideration of momentum, where Eq. (7) will give

$$
\ddot{\lambda}=\ddot{\mu}=\ddot{\theta}=0,
$$

and, thus, a system of order $6\left(C_{0}=-2 C_{2}\right)$,

$$
a \ddot{a}=\frac{C_{0}}{(a b c)^{\gamma-1}}, \quad b \ddot{b}=\frac{C_{0}}{(a b c)^{\gamma-1}}, \quad c \ddot{c}=\frac{C_{0}}{(a b c)^{\gamma-1}} .
$$

The general solution of Eq. (14) would be

$$
\lambda=\lambda_{1} t+\lambda_{0}, \quad \mu=\mu_{1} t+\mu_{0}, \quad \theta=\theta_{1} t+\theta_{0},
$$

where $\lambda_{0}, \lambda_{1}, \mu_{0}, \mu_{1}, \theta_{0}$, and $\theta_{1}$ are constants. For most of the analysis in Sec. III, in order to illustrate the flow physics in a succinct manner, we will look at the degenerate case and will just set, for simplicity,

$$
\lambda=\mu=\theta=0 .
$$

Spherically symmetric flow configurations are then obtained, and precise comparisons with existing works are tabulated in Sec. VI [22-28]. The advantage of the present approach, in contrast to searching directly for spherically symmetric solutions, is that slightly more general three dimensional solutions are possible by taking $\lambda, \mu$, and $\theta$ as linear functions in time $t$, i.e., Eq. (16a).

The system (15) reduces to one form of Ermakov equations for a special value of $\gamma=5 / 3$, i.e., monatomic gas [17,27]. The objective here is to identify a special class of exact solutions of Eq. (15) valid for arbitrary values of $\gamma$, and, thus, new flow configurations of a polyatomic gas will be revealed.

\section{AN EXPANDING SPHERE OF GAS}

Although the analysis for the integrable case of Eq. (15), for $\gamma=5 / 3$ or a monatomic gas, is highly elegant and intriguing, the practical interests might be constrained by these considerations:

(a) Many flows in science and engineering contexts involve diatomic gases, e.g., air.

(b) In many studies of monatomic gas, the Lagrangian formulations are employed for the velocities, which might create an inconvenience in comparing results with a significant portion of the existing literature in fluid mechanics.

\section{A. An exact solution}

The main contribution of the present paper is to recognize a special solution of Eq. (15),

$$
a=b=c=t^{m},
$$

$$
\begin{aligned}
0<m & =\frac{2}{3 \gamma-1} \\
& =\frac{2}{2 \gamma+(\gamma-1)}<\frac{1}{\gamma}<1 \text { if } \gamma>1, \\
& C_{0}=m(m-1) .
\end{aligned}
$$

Consequently, the fluid dynamics variables of velocity, density, and temperature are given by (if $\lambda, \mu$, and $\theta$ are all zero)

$$
\begin{gathered}
\boldsymbol{q}=\frac{m x}{t} \mathbf{i}+\frac{m y}{t} \mathbf{j}+\frac{m z}{t} \mathbf{k}, \\
\rho=\frac{\rho_{0}}{t^{3 m}} \exp \left[+\frac{x^{2}+y^{2}+z^{2}}{t^{2 m}}\right], \\
T=\frac{m(1-m)}{2 t^{2(1-m)}},
\end{gathered}
$$

where $\rho_{0}$ is a constant reference density. The pressure is given by Eq. (5). Equations (19)-(21) constitute an exact solution for the conservation laws of mass and momentum (1) and (2).

Physically, the fluid is flowing radially outward as $m$ is positive, but the speed is decreasing algebraically with respect to time. Given any fixed spherical boundary, the density is an increasing function of spatial coordinates but is a decreasing function of time as a consequence of this outflow. Temperature is uniform throughout space at any fixed instant but also decays as time evolves. In fact, the flow can be regarded as an explosion at time $t=0$ and the subsequent spherical expansion.

\section{B. Analysis of the flow physics}

(a) Vorticity-The flow is devoid of vorticity as a result of the irrotational flow assumption (8), i.e., $\operatorname{curl}(\boldsymbol{q})=\mathbf{0}, \boldsymbol{q}=$ gradient of the scalar potential $\Phi$.

(b) Mass-The total mass of a gas contained in any volume $V$ is given by

$$
\iiint_{V} \rho d x d y d z,
$$

with $\rho$ given by Eq. (20). This can be handled by simple changes of variables,

$$
\xi=\frac{x}{t^{m}}, \quad \eta=\frac{y}{t^{m}}, \quad \zeta=\frac{z}{t^{m}} .
$$

(c) Expansion of a spherical gas cloud-It is instructive to consider a gaseous mass contained initially in the sphere of radius $R_{0}$ at the initial time $t_{0}$,

$$
x^{2}+y^{2}+z^{2}=R_{0}^{2} \quad \text { at } \quad t=t_{0} .
$$

The mass within this sphere is given by

$$
\begin{aligned}
& \rho_{0} \iiint_{x^{2}+y^{2}+z^{2} \leqslant R_{0}^{2}} \exp \left[+\frac{x^{2}+y^{2}+z^{2}}{t^{2 m}}\right] \frac{d x d y d z}{t^{3 m}} \\
& =\rho_{0} \iiint_{t_{0}^{2 m}\left(\xi^{2}+\eta^{2}+\zeta^{2}\right) \leqslant R_{0}^{2}} \exp \left[+\xi^{2}+\eta^{2}+\zeta^{2}\right] d \xi d \eta d \zeta .
\end{aligned}
$$

Due to the outward radial velocities, this gas cloud would have expanded to a new radius $R_{1}$ at time $t_{1}$. The mass within 
this new sphere will be given by an expression similar to Eq. (23) except that the integral is taken over

$$
x^{2}+y^{2}+z^{2}=R_{1}^{2}, \text { or } t_{1}^{2 m}\left(\xi^{2}+\eta^{2}+\zeta^{2}\right)=R_{1}^{2} .
$$

Since all velocity vectors are pointing away from the origin, no fluid particles exterior to this sphere will enter the sphere. Thus, to conserve mass as given by Eq. (23), we must have

$$
\frac{R_{0}}{t_{0}^{m}}=\frac{R_{1}}{t_{1}^{m}},
$$

and this expression determines the new radius $R_{1}$ in terms of the new time $t_{1}$. Consequently, for the mass of gas initially contained in a sphere of $R_{0}$, the radius $R$, at any subsequent time $t$, must satisfy $R \sim t^{m}$.

(iv) Mass flow-The Mach number $M$, ratio of flow speed to the local speed of sound, for the gas on the surface of a sphere $R_{0}$ at time $t_{0}$, Eq. (22), is calculated from Eqs. (19) and (21) as

$$
M^{2}=\frac{2 m R_{0}^{2}}{\gamma(1-m) t_{0}^{2 m}} .
$$

If Eq. (25) holds, when the gaseous particles expand to a radius $R_{1}$ at time $t_{1}$, the Mach number remains constant. This rather surprising conclusion comes from two curious coincidences. First, in a Lagrangian description (following the particle), the velocity decreases with distance. Second, the temperature, related to the square of the sound speed, also drops with time, causing this ratio of flow speed to local sound speed to remain constant.

The mass flux over the surface of this sphere, obtained by integrating the product of density and velocity over an area integral, is

$$
\frac{4 \pi m R_{0}^{3}}{t_{0}^{3 m+1}} \exp \left(+\frac{R_{0}^{2}}{t_{0}^{2 m}}\right) .
$$

For a fixed time $t_{0}$, if one now considers Eq. (27) as a function of the radius $R_{0}$, the mass flux at the surface of a larger sphere will be greater than that of a smaller sphere. This is consistent with the fact that the density will be a decreasing function of time as the outward flux is larger than the influx in such an annular region.

(v) Energy equation-The internal energy $E$ of a gas is given by $E=C_{v} \mathrm{~T}$, where $\mathrm{C}_{p}$ and $C_{v}$ are the specific heat capacities at constant pressure and volume, respectively. In a normalized system where the gas constant is unity,

$$
C_{p}-C_{v}=1, \quad \frac{C_{p}}{C_{v}}=\gamma,
$$

and, thus, one finds

$$
C_{v}=\frac{1}{\gamma-1}, E=C_{v} T .
$$

One very common form for the governing equation of $E$ is

$$
\rho\left[\frac{\partial E}{\partial t}+(\boldsymbol{q} \cdot \boldsymbol{\nabla}) E\right]=-p(\boldsymbol{\nabla} \cdot \boldsymbol{q})+\nabla \cdot(k \boldsymbol{\nabla} T)
$$

where $k$ is the thermal conductivity. For a temperature field dependent on time only, the spatial derivatives will vanish.
One can then readily verify that Eq. (29) is satisfied for Eqs. (5), (19)-(21), and (28).

(vi) Drawback and deficiency-A drawback for the solutions (19)-(21) is that, for any fixed time, the density and velocities increase indefinitely at great distances from the origin. Hence, consideration must be restricted to a finite domain. Second, a temperature field independent of the spatial coordinates will mean the absence of heat conduction. Extension to a wider class of temperature profiles will be left for future studies.

\section{Connection with NLSE}

As a by-product of the whole analysis, an exact solution can be found for the following logarithmic NLSE with a Bohmtype potential:

$$
i \Psi_{t}+\nabla^{2} \Psi-\frac{m(1-m)}{2 t^{2(1-m)}}(\ln |\Psi|) \Psi-\left(\frac{\nabla^{2}|\Psi|}{|\Psi|}\right) \Psi=0 .
$$

This is given by

$$
\begin{aligned}
\Psi= & \frac{\sqrt{\rho_{0}}}{t^{3 m / 2}} \exp \left[+\frac{x^{2}+y^{2}+z^{2}}{2 t^{2 m}}\right. \\
& \left.+\frac{i m}{4 t}\left(x^{2}+y^{2}+z^{2}\right)+i G(t)\right] .
\end{aligned}
$$

This solution is different from the gausson as there are both algebraic and exponential components contributing to the magnitude of the complex envelope $\Psi$, resulting from a Bohmtype nonlinearity $\Delta|\Psi| /|\Psi|$ in the governing equation (30) in addition to the logarithmic potential. For system (30) and solution (31), $m$ is a free parameter. The function $G(t)$ arises from the time dependence of the velocity potential and has no direct physical consequence in terms of fluid velocity and density. It is determined by direct substitution into Eq. (30) and equating terms involving only time.

\section{PERSPECTIVES FROM CLASSICAL GAS DYNAMICS}

It will be instructive to study this class of exact solutions from the perspective of classical fluid mechanics.

\section{A. Density gradient and vorticity}

In dynamic meteorology, it is not uncommon to have vorticity generated from a density gradient. The general vorticity equation for a fluid with variable density is $(\omega$ is the vorticity vector)

$$
\left(\frac{\partial}{\partial t}+\boldsymbol{q} \cdot \nabla\right) \frac{\boldsymbol{\omega}}{\rho}=\left(\frac{\boldsymbol{\omega}}{\rho} \cdot \nabla\right) \boldsymbol{q}+\frac{1}{\rho^{3}}(\nabla \rho \times \nabla p) .
$$

In the present case of an ideal gas $(p=\rho T)$ with temperature being a function of time $t$ only, the spatial gradients of $p$ and $\rho$ are parallel, and the vector product is zero. Hence, a flow with zero vorticity initially will remain irrotational.

\section{B. One dimensional gas dynamics}

The 1D version of Sec. III deserves further study as the algebraic complexity reduces considerably. In fact, the whole 
expansion scheme still works, provided we neglect terms associated with the higher dimensions. For the 1D case, Eq. (15) degenerates to

$$
a \ddot{a}=\frac{C_{0}}{a^{\gamma-1}},
$$

i.e., one equation for $a(t)$ with $b(t)$ and $c(t)$ absent [or setting $b(t)$ and $c(t)$ to be unity]. The other fluid properties are given by [with Eq. (14), still holds]

$$
\begin{gathered}
\rho=\frac{\rho_{0}}{a} \exp \left[\frac{(x-\lambda)^{2}}{a^{2}}\right], \\
u=\frac{\dot{a}}{a}(x-\lambda)+\dot{\lambda} .
\end{gathered}
$$

Along the line of reasoning of Eq. (17), a solution for Eq. (32) is

$$
a(t)=t^{2 /(\gamma+1)} .
$$

This set of solutions will be employed to study a 1D expansion of a gas column in the next section.

\section{Unsteady potential flow in two dimensions}

A compressible irrotational flow will have a highly nonlinear governing equation for the velocity potential $[1,19,20]$. It will be illuminating to present an unsteady version first. The solution scheme in the present paper will then be utilized to derive an exact solution for this complicated evolution equation.

We first start with the $x$-momentum equation and Eq. (5) (a similar situation will hold for the $y$ direction, $c_{\text {sound }}=$ speed of sound),

$$
\frac{\gamma}{c_{\text {sound }}^{2}}\left(u_{t}+u u_{x}+v u_{y}\right)=-\frac{\rho_{x}}{\rho} .
$$

With the assumption of irrotational flow (8), integration in spatial coordinates will give

$$
\frac{\gamma}{c_{\text {sound }}^{2}}\left(\Phi_{t}+\frac{\Phi_{x}^{2}+\Phi_{y}^{2}}{2}\right)=-\ln (\rho) .
$$

The arbitrary function of time in this integration will be accounted for later by incorporating this arbitrary function in the velocity potential.

Differentiation of this equation for $\ln (\rho)$ with respect to $t$, $x$, and $y$ and substitution into the continuity equation,

$$
\rho_{t}+u \rho_{x}+v \rho_{y}+\rho\left(u_{x}+v_{y}\right)=0,
$$

will give (on canceling the common factor $\rho$ )

$$
\begin{aligned}
\frac{1}{\gamma}\left(\Phi_{x x}+\Phi_{y y}\right)= & {\left[\frac{1}{c_{\text {sound }}^{2}}\left(\Phi_{t}+\frac{\Phi_{x}^{2}+\Phi_{y}^{2}}{2}\right)\right]_{t} } \\
& +\Phi_{x}\left[\frac{1}{c_{\text {sound }}^{2}}\left(\Phi_{t}+\frac{\Phi_{x}^{2}+\Phi_{y}^{2}}{2}\right)\right]_{x} \\
& +\Phi_{y}\left[\frac{1}{c_{\text {sound }}^{2}}\left(\Phi_{t}+\frac{\Phi_{x}^{2}+\Phi_{y}^{2}}{2}\right)\right]_{y} .
\end{aligned}
$$

In the limit of an incompressible fluid ( $c_{\text {sound }} \rightarrow \infty$ ), the Laplace equation is recovered.

To solve for a two dimensional flow along the theme of this paper, the governing differential equations are still Eqs. (11)(13) and (15) but with the $z$ direction terms involving $c(t)$ absent. The expression for $a(t)$ is $a\left(d^{2} a / d t^{2}\right)=C_{0} a^{2(\gamma-1)}$, i.e.,

$$
a=t^{1 / \gamma} .
$$

The velocity vector is

$$
q=[x /(\gamma t)] \mathbf{i}+[y /(\gamma t)] \mathbf{j} .
$$

The potential easily is deduced to be

$$
\Phi=\left[\left(x^{2}+y^{2}\right) /(2 \gamma t)\right]+g(t),
$$

where $g(t)$ measures the time dependence of the potential and has no direct physical significance in terms of the velocity or density. The speed of sound is given by (gas constant has been normalized to unity)

$$
c_{\text {sound }}^{2}=\gamma T=(\gamma-1) / Y, \quad Y=2 \gamma t^{2(\gamma-1) / \gamma} .
$$

Substituting Eq. (36) into Eq. (35) shows that the $\left(x^{2}\right.$ $\left.+y^{2}\right)$ terms on both sides match exactly, while $g(t)$ is defined by the terms involving only time $t$. Thus, the present family of solutions can provide a simple exact solution for the unsteady potential Eq. (35), with extensions to other dimensions conceptually straightforward.

\section{ONE DIMENSIONAL GAS FLOW}

The present formulation can be employed to produce an exact description for a 1D, ideal gas flow in a finite domain. Consider a gas, with $\gamma$ again being the ratio of specific heat capacities, confined between a fixed wall on the left $(x=0)$, and a right boundary that is moving further to the right at a given, but otherwise arbitrary, velocity. New fluid is being injected at the fixed wall with a constant speed. Equations (32)-(34) with $\lambda=V_{0} t, V_{0}$ constant, will give an exact description of this scenario as

$$
\begin{gathered}
0<x<V_{0} t, t>0, \\
\rho=\frac{\rho_{0}}{t^{2 /(\gamma+1)}} \exp \left[\frac{\left(x-V_{0} t\right)^{2}}{t^{4 /(\gamma+1)}}\right],
\end{gathered}
$$

$$
T=(\gamma-1) /\left[(\gamma+1)^{2} Z\right], \quad Z=t^{2(\gamma-1)(\gamma+1)}, \quad p=\rho T,
$$

$$
\begin{aligned}
u & =\frac{2}{\gamma+1}\left(\frac{x-V_{0} t}{t}\right)+V_{0} \\
& =\frac{2 x}{(\gamma+1) t}+\left(\frac{\gamma-1}{\gamma+1}\right) V_{0} .
\end{aligned}
$$

Obviously, the apparent singularity $t=0$ must be excluded from consideration, and this solution is only applicable for $t>0$. In physical terms, the two walls coincide or are at an 


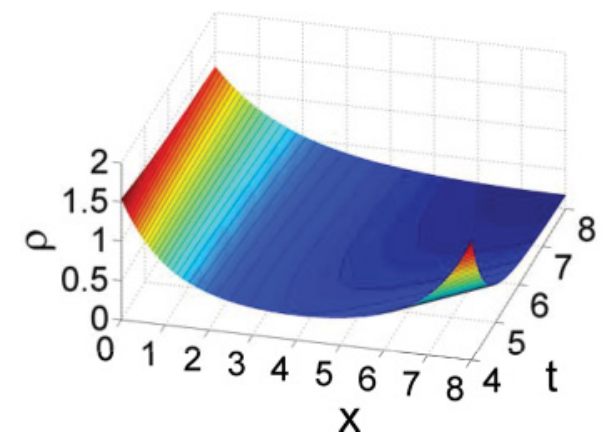

FIG. 1. (Color online) A three dimensional plot for the density $\rho$ of Eq. (37) versus $x$ and $t, \rho_{0}=V_{0}=1$. The density field is well defined for all values of $x$ and $t$. However, if the gas column model of $\mathrm{Sec} . \mathrm{V}$ is adopted, the fluid is confined in the interval $0<x<V_{0} t$.

infinitesimal distance apart at $t=0$. Fluid is injected at a speed of $(\gamma-1) V_{0} /(\gamma+1)$ (which is $u$ at $\left.x=0\right)$ into the domain $0<x<V_{0} t$. The boundary on the right side is translated uniformly further to the right starting at $t=0$. The fluid speed at $x=V_{0} t$ is $V_{0}$ too, and, thus, the normal velocities of the fluid and the wall are identical. The density, which is defined for all $x$ and $t$, is illustrated in Fig. 1. The minimum will occur at $x=V_{0} t$. Thus, in the domain $0<x<V_{0} t$, the density is a monotonically decreasing function with the highest density at the left boundary, the site of fluid injection.

A particularly illuminating physical quantity to study is the mass confined within this gaseous expansion. From first principles, for a $1 \mathrm{D}$ gas flow, the mass in the domain $0<x<$ $V_{0} t$ is

$$
\int \rho(x, t) d x
$$

where the lower and upper limits should be 0 and $V_{0} t$, respectively. Using Eq. (37) and defining $z=x-V_{0} t$, this mass is

$$
\int_{0}^{V_{0} t} \rho d x=\int_{0}^{V_{0} t} \frac{\rho_{0}}{t^{2 /(\gamma+1)}} \exp \left(\frac{z^{2}}{t^{4 /(\gamma+1)}}\right) d z .
$$

On using a new variable,

$$
\xi=\frac{z}{t^{2 /(\gamma+1)}}
$$

this mass is

$$
\int_{0}^{\Omega} \rho_{0} \exp \left(\xi^{2}\right) d \xi, \text { where } \Omega=V_{0} t^{(\gamma-1) /(\gamma+1)} .
$$

Next, we are going to show that this is exactly the same mass resulting from the influx at the left wall. The density $[\rho(x, t)]$ and velocity $[u(x, t)]$ are functions of $x$ and $t$, Eqs. (37) and (38). The mass flux generally is given by the density multiplied by the velocity. Consequently, the total mass injection at the left wall, from $t=0$ up to time $t$, is

$$
\int_{0}^{t} \rho(0, \tau) u(0, \tau) d \tau
$$

From Eqs. (37) and (38), this expression is

$$
\int_{0}^{t} \frac{\rho_{0}}{\tau^{2 /(\gamma+1)}} \exp \left[V_{0}^{2} \tau^{2(\gamma-1) /(\gamma+1)}\right]\left(\frac{\gamma-1}{\gamma+1}\right) V_{0} d \tau \text {. }
$$

By a simple change of variable,

$$
V_{0} \tau^{(\gamma-1) /(\gamma+1)}=\eta
$$

this is the same as Eq. (39). Consequently, mass is conserved.

Thus, we can conclude that this theory can provide an exact formulation of a gaseous expansion due to a constant mass flux at a fixed wall, while the other wall is allowed to move at a constant velocity.

\section{CONCLUSIONS AND DISCUSSIONS}

A class of exact solutions for irrotational, isentropic, and compressible flows is obtained by special expansion schemes of the fluid properties. Physically, the density is Gaussian in terms of a similarity variable, while the temperature is a function of time only. The velocity depends linearly on the spatial coordinates. The present class of solutions is valid for any ratio of the specific heat capacities of the gas. Thus, this extends many elegant and highly intriguing works in the literature that treated the monatomic (i.e., integrable) case only.

The present algorithm is applicable to $1 \mathrm{D}$, two, and three dimensions. As an illustrative example, an analytical description of a 1D gas flow with mass injection through one boundary is possible. From the perspective of classical fluid mechanics, this approach, in terms of similarity variables, provides an exact solution for the velocity potential in unsteady, inviscid, irrotational, and compressible flows.

Employing the context of gas clouds in spherical geometry, a remark on the quantitative comparisons between existing works and this paper is in order. Earlier works usually made one or more of these assumptions:

(1) polynomial [22-25] or exponential density distributions [26],

(2) monatomic gas $(\gamma=5 / 3)[17,27]$, or

(3) slab geometry [23].

We also verify that our results are different from several existing ones in the dynamics of a spherical gas cloud [28]. Consequently, the results here are different from those in the literature.

Nevertheless, there are several points where this class of exact solutions might need further refinement and improvement. First, the velocity and density increase indefinitely with distance from the origin, and, hence, a finite domain must be considered. Indeed, exact solutions with an infinite amount of energy do exist. A well known example is the Burgers vortex in a straining flow [29-31], which models the dynamics of vortex tubes in turbulence. Quantitative comparisons with the present paper are difficult, as we deal with inviscid, irrotational, and compressible flows here. None of these conditions are applicable to the Burgers vortex.

Second, the lack of a spatial derivative for the temperature for the present class of solutions implies that heat conduction cannot be important in this model. Finally, another potential application of the present formulation is in stellar dynamics and astrophysics, where gravitational effects might have to be restored. Extensions to other fields of physics would also be valuable. In fact, a similar solution with a Gaussian density has also been employed in laser and plasma studies [32,33]. 
However, the physical contexts there are different, with the focus on the energy equation and heat effects of the lasers.

Exact solutions are rare in compressible flows [34-36], and, in fact, for fluid mechanics in general. Hence, results obtained in this paper can provide valuable physical insight and may serve as benchmarks for testing numerical codes.

\section{ACKNOWLEDGMENTS}

The author would like to thank Professor Colin Rogers for introducing this subject to his research group. Partial financial support is provided by the Research Grants Council Contract No. HKU 7120/08E.
[1] I. G. Currie, Fundamental Mechanics of Fluids (McGraw Hill, New York, 1993).

[2] A. Bisighini, G. E. Cossali, C. Tropea, and I. V. Roisman, Phys. Rev. E 82, 036319 (2010).

[3] D. R. Lester, G. Metcalfe, M. G. Trefry, A. Ord, B. Hobbs, and M. Rudman, Phys. Rev. E 80, 036208 (2009).

[4] S. C. Anco and A. Dar, Proc. R. Soc. London, Ser. A 466, 2605 (2010).

[5] T. Funada, D. D. Joseph, M. Saitoh, and S. Yamashita, Int. J. Multiphase Flow 32, 20 (2006).

[6] J. Li, SIAM J. Appl. Math. 62, 831 (2001).

[7] D. W. Moore and D. I. Pullin, J. Fluid Mech. 374, 285 (1998).

[8] I. Bialynicki-Birula and J. Mycielski, Phys. Scr. 20, 539 (1979).

[9] G. Lauro, Geophys. Astrophys. Fluid Dyn. 102, 373 (2008).

[10] S. De Martino, M. Falanga, C. Godano, and G. Lauro, Europhys. Lett. 63, 472 (2003).

[11] E. F. Hefter, Phys. Rev. A 32, 1201 (1985).

[12] W. Krolikowski, D. Edmundson, and O. Bang, Phys. Rev. E 61, 3122 (2000).

[13] T. Hansson, D. Anderson, and M. Lisak, Phys. Rev. A 80, 033819 (2009).

[14] J. L. Reid and J. R. Ray, J. Math. Phys. 21, 1583 (1980).

[15] C. Rogers and W. K. Schief, J. Math. Anal. Appl. 198, 194 (1996).

[16] C. Rogers, B. A. Malomed, K. Chow, and H. An, J. Phys. A 43, 455214 (2010).

[17] B. Gaffet, J. Fluid Mech. 325, 113 (1996).

[18] W. C. Thacker, J. Fluid Mech. 107, 499 (1981).
[19] C. S. Yih, Fluid Mechanics: A Introduction of the Theory (McGraw Hill, New York, 1969).

[20] J. D. Anderson, Modern Compressible Flows: with Historical Perspective (McGraw Hill, New York, 1990).

[21] A. B. Nassar, Phys. Rev. A 33, 3502 (1986).

[22] J. P. Vishwakarma and A. K. Singh, J. Astrophys. Astron. 30, 53 (2009).

[23] C. Boudesocque-Dubois, S. Gauthier, and J. M. Clarisse, J. Fluid Mech. 603, 151 (2008)

[24] A. I. Shestakov, Phys. Fluids 11, 1091 (1999).

[25] V. Simonsen and J. Meyer-ter-Vehn, Phys. Fluids 9, 1462 (1997).

[26] K. K. Singh and J. P. Vishwakarma, J. Theor. Appl. Mech. 45, 801 (2007).

[27] A. Donato and T. Ruggeri, J. Math. Anal. Appl. 251, 395 (2000).

[28] P. L. Sachdev, K. T. Joseph, and M. Ejanul Haque, Stud. Appl. Math. 114, 325 (2005).

[29] P. G. Saffman, Vortex Dynamics (Cambridge University Press, New York, 1992).

[30] K. Higgins, M. Rütten, A. Ooi, and M. S. Chong, Fluid Dyn. Res. 41, 035502 (2009).

[31] T. Gallay and Y. Maekawa, Commun. Math. Phys. 302, 477 (2011).

[32] J. H. Hunter and R. A. London, Phys. Fluids 31, 3102 (1988).

[33] L. Ferrario, J. Plasma Phys. 64, 1 (2000).

[34] J. G. Wouchuk, C. Huete Ruiz de Lira, and A. L. Velikovich, Phys. Rev. E 79, 066315 (2009).

[35] G. E. Crooks and C. Jarzynski, Phys. Rev. E 75, 021116 (2007).

[36] A. R. Manwell, Wave Motion 2, 83 (1980). 